\title{
Morbidity and Mortality after Colorectal Surgery for Cancer
}

\author{
Giulio Paolo Angelucci ${ }^{*}$, Giovanni Sinibaldi ${ }^{2}$, Paolo Orsaria ${ }^{1}$, Claudio Arcudi ${ }^{1}$, Sergio Colizza ${ }^{2}$ \\ ${ }^{1}$ University of Rome “Tor Vergata”, Policlinico Tor Vergata, Surgery, Rome, Italy \\ ${ }^{2}$ Hospital San Giovanni Calibita Fatebenefratelli, Isola Tiberina, Surgery, Rome, Italy \\ Email: "giuliopangelucci@gmail.com
}

Received October 31, 2013; revised November 19, 2013; accepted November 25, 2013

Copyright (c) 2013 Giulio Paolo Angelucci et al. This is an open access article distributed under the Creative Commons Attribution License, which permits unrestricted use, distribution, and reproduction in any medium, provided the original work is properly cited.

\begin{abstract}
Colorectal Cancer is the second most common cancer in western countries and, currently, surgical resection is still the principal treatment for this pathology. However, the operation carries significant morbidity and mortality, which is associated with an enormous use of healthcare resources. The aim of our study is to evaluate the incidence and the management of complications, and to understand how pre-exisiting comorbidities can influence the recovery of the patients. Between 2007 and 2012, a total of 534 patients underwent elective or emergency surgery for colorectal cancer in our department. Patients were identified for this study from a prospectively entered computerized database. Case notes of patients documented comorbidities, type of surgery performed, complication in the early postoperative period (30 days after surgery) and the management. Postoperative morbidity affected 89 patients (17\%), of these 25 (27\%) were anastomotic leakage (AL). 22 (24\%) patients experienced intra-abdominal abscess. 16 patients (17\%) had wound infections. 11 patients (13\%) experienced post-operative bleeding and five of them had a re-operation within the I and the II day after surgery. 12 (13\%) complained medical (cardiologic/respiratory) complications. We had 1 (1\%) Small Bowel Obstruction, treated with a conservative therapy. Reoperation rate was 3\% with 11 for AL, and 5 for bleeding. The mortality rate was $0.55 \%$ (3 patients). In our experience, we evidenced that surgery performed for advanced rectal cancer in the lower rectum, especially in urgency settings is associated with an increase of morbidity and mortality in the early post-operative period. Pre-existing comorbidities are involved in the morbidity of the patients, and a more accurate approach both in surgical technique and in the post-operative management can be proposed to the surgeon. Derivative stoma in high risk patients gave us the possibility of a conservative treatment of the Anastomotic Leak, the most common complication in our study, with antibiotics and CT-drainage.
\end{abstract}

Keywords: Colorectal; Colorectal Surgery; Morbidity; Mortality; Surgery; Complications; Early Post-Operative Complication; Management; Anastomotic Leak; Leakage

\section{Introduction}

Colorectal Cancer is the second most common cancer in western countries [1] and, currently, surgical resection is still the principal treatment for this pathology. The operation carries significant morbidity which is associated with an enormous use of healthcare resources, and mortality between $1 \%$ and $2 \%$ [2].

Anastomotic Leak (AL) is the most fearful complication by the colorectal surgeon. The reported rate of colonic anastomotic leak is in the range from $1.5 \%$ up to $16 \%$, with mortality ranging between $10 \%$ and $20 \%$ [2,3]. Total Mesorectal Excision (TME) which is mandatory for the treatment of carcinoma of the lower and mid rectum

${ }^{*}$ Corresponding author. is associated with an increased risk of clinical anastomotic leakage [4]. In addition, patients who experienced a complication in the early postoperative period have been demonstrated to have poorer long-term functional results, increased local recurrence rate and reduced 5-year cancer survival [5].

The aim of our study is to evaluate the incidence and the management of complications, and to understand how pre-existing comorbidities can influence the recovery of the patients.

\section{Methods}

Between 2007 and the 2012 a total of 534 patients underwent elective or emergency surgery for colorectal 
cancer in our department; 68 (13\%) of these were operated using laparoscopic approach. Patients were identified for this study from a prospectively entered computerized database. Case notes of patients documented comorbidities, type of surgery performed, complications in the early postoperative period (30 days after surgery) and its management.

Age, gender, physical status and comorbidities were evaluated (Table 1).

Antibiotics prophylaxis was given with intravenous administration for a mean time of three days, using Piperacillin and Gentamycin.

The indication for surgery was malignant disease as rectal cancer in 161 patients, colonic cancer in 368 patients. 5 patients underwent total colectomy for Familial Adenomatous Polyposis. The distribution of Duke's stages was: A (invasion into but not through the bowel wall) 105 (19\%), B (invasion through the bowel wall but not involving lymph nodes) 150 (28\%), C (involvement of lymph nodes) 183 (34\%). 84 patients (13\%) had benign disease or incomplete staging.

Surgical procedures were: 190 Right Colectomies, 177 Left Colectomies, 162 Low Anterior Resection, 5 Total Colectomies. Patients who had only explorative laparotomy or laparoscopy were excluded from the study.

The decision in favour of laparoscopic approach was based on surgeon experience, patient's characteristics, tumor size, and tumor staging. Drains were always used for 2 - 3 days.

The great part of the anastomoses was made by stapler, except for few right haemicolectomies. Oral fluid and food intake was started gradually between the second and the third day after surgery.

The definition of an anastomotic leakage was clinical: peritonitis caused by leakage, pelvic abscess, pus or gas from the abdominal drain. All anastomotic leakage was confirmed by one or more of the following: CT scan, Barium Enema, and reoperation.

Incidence of wound infections, Small Bowel Obstruction (SBO), postoperative bleeding, abdominal abscess

Table 1. Patients' demography.

\begin{tabular}{cc}
\hline $\begin{array}{c}\text { Number of patients 534 } \\
\text { Age }\end{array}$ & Male 282 (53\%) Female 252 (47\%) \\
Mean hospital stay & 11.6 days, range 1 - 71, SD 8.4 \\
& 190 Right Colectomies \\
Type of surgery & 177 Left Colectomies \\
& 162 Low Anterior Resection \\
& 5 Total Colectomies \\
Comorbidities & 86 pts (16\%) \\
& Cardiovascular 68 \\
Diabetes 16 \\
Approach & Others 2 \\
& Open $466(87 \%)$ \\
\end{tabular}

and medical complication were also recorded.

Statistical analysis was made using Fisher's exact test.

\section{Results}

The total number of patients who underwent Colorectal Surgery (CRS) in our department was 618 (Male 316 51\%/Female 302 48.9\%), the mean age was 68.1 (SD 11.3). Of these, 534 patients underwent elective or emergency surgery for CRS for cancer. Mean Hospital stay was 11. 6 days, SD 8.4.

Postoperative morbidity affected 89 patients (17\%), 25 of these $(27 \%)$ were anastomotic leakage, occurring with in the III and the IX day after surgery. 4 of these patients were re-operated with laparoscopic technique, 7 with open surgery (Table 2).

22 (24\%) patients experienced intra-abdominal abscess usually treated with intra venous antibiotics administration and CT-Scan monitoring. In 4 of them, CT drainage was performed.

16 out of 89 patients (17\%) had wound infections. 11 patients (13\%) experienced post-operative bleeding and five of them a re-operation within the I and the II day after surgery. 12 (13\%) complained medical (cardiologic/respiratory) complications, one patient of these died in the immediate postoperative period. 2 patients (2\%) complained ileus treated with conservative therapy. We had 1 (1\%) Small Bowel Obstruction, treated with a conservative therapy (Table 3).

16 patients (17\% of the complicated patients) underwent laparotomy for surgical complications: 11 for anastomotic leakage, between the III and IX day after surgery mean time (5.1 SD 2.4). 5 patients underwent reoperation for bleeding the same day after the procedure or the day after (Table 4). We also evaluated the Duke's stage related complications since the tumor invasion could be associated with an increased risk of $\mathrm{AL}$, as reported in literature [1,2], however, in our study there was not a statistical evidence (Table 5).

Otherwise we found correlation between pre-existing comorbidities and complications rate to be statistically significant $(\mathrm{p}=0.00775)$.

\section{Discussion}

In this population based study of 534 patients operated

Table 2. Anastomotic leakage and mortality.

\begin{tabular}{ccccc}
\hline & Total num. & Number of & & \\
& procedures & leaks & Leak rate (\%) Mortality \\
\hline Anterior Resection & 162 & 12 & $7 \%$ & $2(1.2 \%)$ \\
L Hemicolectomy & 177 & 5 & $3 \%$ & \\
R Hemicolectomy & 190 & 7 & $4 \%$ & $1(0.5 \%)$ \\
Total Colectomy & 5 & 1 & $20 \%$ & \\
Total & 534 & 25 & $4.7 \%$ & $3(0.55 \%)$ \\
\hline
\end{tabular}


Table 3. Overall morbidity.

\begin{tabular}{cc}
\hline Complications & $\mathrm{n}$ \\
\hline Anastomotic Leakage & $25(4.7 \%)$ \\
Intra-Abdominal Abscess & $22(4.1 \%)$ \\
Wound Infections & $16(3 \%)$ \\
Bleeding & $11(2 \%)$ \\
Cardiological/Respiratory & $12(2 \%)$ \\
Ileus & $2(0.3 \%)$ \\
SBO & $1(0.2 \%)$ \\
Total & $89 / 534(17 \%)$ \\
\hline
\end{tabular}

Table 4. Re-operation rate and morbidity.

\begin{tabular}{lcc}
\hline & Bleeding & $\mathrm{AL}$ \\
\hline Reoperations (3\%) & 5 & 11 \\
\hline
\end{tabular}

Table 5. TNM related complications.

Duke stage A: $19 \%$ experienced AL
Duke stage B: $28 \%$ experienced AL
Duke stage C: $34 \%$ experienced AL

for colorectal cancer 89 (17\%) experienced a complication. $25(28 \%)$ of them had an anastomotic leakage, a total of $4.7 \%$ with an associated mortality of $0.55 \% .12$ of them after an anterior resection and a TME. One well recognized risk factor is shown to be the level of the anastomosis, usually between $7 \mathrm{~cm}$ and $5 \mathrm{~cm}$ from the anal verge [6]. In present study low anastomosis was an independent risk factor. In $69 \%$ of cases the reoperation performed was laparotomy, drainage and stoma formation. In 58 out of 162 patients treated by anterior resection of the rectum had a contemporary right colostomy (very rarely, Ileostomy). Such approach was usually limited to patients who had a primary radio-chemotherapy. In case of AL, these patients were cured by CT-scan drainage (8 cases), even though the role of temporary stoma is still controversial [7].

In our experience, the major advantage was a less dramatic consequences when the leak occurs, giving us the possibility of a conservative treatment. Disadvantages were the cost of a second operation and the possible stoma related complications.

Identifying possible risk factor for an anastomotic leakage could be useful to select patients who may need a temporary stoma. Pre-existing comorbidities (cardiorespiratory), reduced preoperative nutritional status (anemia, hypoprotidedemia and weight loss), set of surgery (emergency and elective) previous radiation and gender (male > risk) [8] were recorded as a guide for intra-operative decisions in our experience.

Previous studies concluded that significant clinical indicators of leakage were fever $\left(>38^{\circ} \mathrm{C}\right)$ on day 2 , absence of bowel action on day 4, diarrhea before day 7, more than $400 \mathrm{ml}$ of fluid in the abdominal drain by day 3, renal failure on day 3 and leukocytosis on day 7 [7]. However, our analysis shows that the majority of patients who experienced an anastomotic leakage had a radiological diagnosis. The decision of a reoperation was usually made on the data from the CT scanning with rectal contrast associated on the clinical findings.

4 of the 68 (13\%) patients who underwent a colonic resection using laparoscopic technique experienced an anastomotic leak. Such figure is greater than the mean incidence (4.7\%) but it is not statistically significant. We can not also demonstrate a reduction of complication such bleeding, wound infection and medical complications in short term postoperative outcome.

Intra-abdominal abscess were the second most frequent complication that our patients experienced. According with the literature the treatment consisted in percutaneous CT-guided abscess drainage that is shown to be an effective method for treating intra-abdominal abscess following elective colorectal surgery with a range of success between $65 \%$ after the first and $85 \%$ after a second attempt [9].

Postoperative wound infection occurred in 16 (3\%) of the patients. In contaminated surgery, such as elective major colorectal surgery, has a reported incidence of incisional Surgical Site Infections (SSI), ranging from 10\% - $15 \%$ to $20 \%$ - $25 \%$ associated with significant morbidity, prolonged hospital length of stay, and a high cost to the patient and the institution [10]. In our experience intravenous antibiotics were given 30 minutes before the surgery, as suggested in literature. Preoperative cleaning of the patient's skin was made by chlorhexidine-alcohol. It is shown that is superior to povidoneiodine for preventing surgical-site infection after contaminated surgery, including colorectal procedures [11]. Wound Infection occurred more frequently in emergency surgery. Two cases after perforation of the colon for a large bowel obstruction due to advanced cancer. Five patients were diabetics and obese. The treatment of the wound infection was based on out-patient clinic medications and in three cases with a prolonged antibiotic therapy.

16 patients (3\%) underwent a reoperation, five for an immediate bleeding after surgery, and eleven for an anastomotic leakage, two of these died after the reoperation. In literature the data regarding reoperation after colorectal surgery is variable. In a large series of patients the re-operation rate is shown to be up to $12.5 \%$ and the approach is performed with laparotomy between the $60 \%$ and the $85 \%$ of the patients [8]. However, the limit in our study to compare these results is evident for the great number of patients undergoing a right haemicolectomy, so with a lower risk of anastomotic leakage and without the risk of the mobilization of the splenic flexure.

12 patients experienced medical complications. The management of colorectal surgical patients requires me- 
ticulous fluid and electrolyte therapy, and the involvement of a multidisciplinary team comprehensive of anesthesiologist for the understanding of the pre-existing comorbidities that are involved in the morbidity of the patients [12]. Age was non-related with the morbidity and mortality. Patients with ASA grade $>2$, or cardiovascular disease and/or respiratory disease had higher incidence of morbidity and experienced longer recovery in Intensive Care Unit. Four of them died for heart failure, and one for respiratory failure.

It is also demonstrated a significant ongoing risk of SBO after colorectal surgery, mainly during the 1st year after surgery. It is usually associated with the adhesions formation, especially after open surgery [13]. We had two cases of postoperative ileus and one patient experienced a SBO treated with no oral intake, gastric tube and fluid therapy. The diagnosis of SBO was based on clinical and imaging criteria. Current practice for management of SBO is to give patients who are clinically stable and without evidence of bowel ischemia or strangulation a trial of conservative management. Previously published data suggest that $43 \%$ to $70 \%$ of these patients have resolution of their SBO [14].

\section{Conclusions}

Our study is a retrospective case review, focusing on how comorbidities can influence the outcome of the patients and on the management of the surgical complication. The limit of our clinical records is related to the short-term follow-up, the number of the patients, and the few patients treated with a laparoscopic approach.

In our experience, we evidenced that surgery performed for advanced rectal cancer in the lower rectum, especially in urgency settings is associated with an increase of morbidity and mortality in the early post-operative period, higher than colonic resections.

Pre-existing comorbidities are involved in the morbidity of the patients: obesity, diabetes, cardiovascular disease, respiratory disease and renal failure. And a more accurate approach both in surgical technique and in post-operative management can be proposed to the surgeon. In our experience, age per-se is not a serious independent risk factor unless the patient has one of the previous comorbidities.

It seems that derivative stoma in high risk patients does not decrease the incidence of AL, but may give us the possibility of a conservative treatment with antibiotics and CT-drainage.

\section{REFERENCES}

[1] La Ries, "The Annual Report to the Nation on the Status of Cancer, 1973-1997," Cancer, Vol. 88, No. 10, 2000, pp. 2390-2424.
[2] N. C. Buchs, et al., "Incidence Consequences and Risk Factors for Anastomotic Dehiscence after Colorectal Surgery: A Prospective Monocentric Study,” International Journal of Colorectal Disease, Vol. 23, No. 3, 2008, pp. 265-270. http://dx.doi.org/10.1007/s00384-007-0399-3

[3] J. A. Smith, et al., "Evidence of the Effect of Specialization on the Management, Surgical Outcome and Survival from Colorectal Cancer in Wessex," British Journal of Surgery, Vol. 90, No. 5, 2003, pp. 583-592.

http://dx.doi.org/10.1002/bjs.4085

[4] R. J. Aitken, "Mesorectal Excision for Rectal Cancer," British Journal of Surgery, Vol. 83, No. 2, 1996, pp. 214216. http://dx.doi.org/10.1002/bjs.1800830218

[5] G. Branagan, "Prognosis after Anastomotic Leakage in Colorectal Surgery," Diseases of the Colon \& Rectum, Vol. 48, No. 5, 2005, pp. 1021-1026. http://dx.doi.org/10.1007/s10350-004-0869-4

[6] N. D. Karanjia, "Risk of Peritonitis and Fatal Septicemia and the Need to Defuncion the Low Anastomosis," British Journal of Surgery, Vol. 78, No. 2, 1991, pp. 196-198. http://dx.doi.org/10.1002/bjs.1800780221

[7] A. Alves, "Management of Anastomotic Leakage after Nondiverted Large Bowel Resection," Journal of the American College of Surgeons, Vol. 189, No. 6, 1999, pp. 554-559. http://dx.doi.org/10.1016/S1072-7515(99)00207-0

[8] P. Matthiessen, "Risk Factors for Anastomotic Leakage after Anterior Resection of the Rectum," Colorectal Disease, Vol. 6, No. 6, 2004, pp. 462-469. http://dx.doi.org/10.1111/j.1463-1318.2004.00657.x

[9] M. Khurrum Baig, "Percutaneous Postoperative IntraAbdominal Abscess Drainage after Elective Colorectal Surgery," Techniques in Coloproctology, Vol. 6, No. 3, 2002, pp. 159-164.

http://dx.doi.org/10.1007/s101510200036

[10] R. Tang, "Risk Factors for Surgical Site Infection after Elective Resection of the Colon and Rectum: A Single-Center Prospective Study of 2809 Consecutive Patients," Annals of Surgery, Vol. 234, No. 2, 2001, pp. 181-189.

http://dx.doi.org/10.1097/00000658-200108000-00007

[11] R. O. Darouiche, "Chlorhexidine-Alcohol versus Povidone-Iodine for Surgical-Site Antisepsis,” New England Journal of Medicine, Vol. 362, No. 1, 2010, pp. 18-26. http://dx.doi.org/10.1056/NEJMoa0810988

[12] P. Santosh, et al., "Anesthesia and Perioperative Management of Colorectal Surgical Patients," Journal of Anaesthesiology Clinical Pharmacology, Vol. 28, No. 3, 2012, pp. 304-313.

http://dx.doi.org/10.4103/0970-9185.98321

[13] G. Miller, "Natural History of Patients with Adhesive Small Bowel Obstruction,” British Journal of Surgery, Vol. 87, No. 9, 2000, pp. 1240-1247. http://dx.doi.org/10.1046/j.1365-2168.2000.01530.x

[14] M. C. Parker, et al., "Postoperative Adhesions: Ten-Year Follow-Up of 12,584 Patients Undergoing Lower Abdominal Surgery,” Diseases of the Colon \& Rectum, Vol. 44, No. 6, 2001, pp. 822-829. http://dx.doi.org/10.1007/BF02234701 


\section{Abbreviations}

AL: Anastomotic Leak;

TME: Total Mesorectal Excision;

SBO: Small Bowel Obstruction;

CRS: Colorectal Surgery;

SSI: Surgical Site Infections. 